\title{
A new miracidia hatching device for diagnosing schistosomiasis
}

\author{
Arnon D Jurberg, Áureo A de Oliveira ${ }^{1}$, Henrique L Lenzi, Paulo Marcos Z Coelho ${ }^{1,2}$ + $^{+}$ \\ Laboratório de Patologia, Instituto Oswaldo Cruz-Fiocruz ${ }^{1}$ Laboratório de Esquistossomose, Instituto de Pesquisa René Rachou-Fiocruz, Av. \\ Augusto de Lima 1715, 30190-002 Belo Horizonte, MG, Brasil ²Santa Casa de Misericórdia de Belo Horizonte, Belo Horizonte, MG, Brasil \\ It is still imperative to develop a parasitological technique highly sensitive for diagnosing schistosomiasis in \\ epidemiological and individual surveys. A simple and cheap hatching device with a collecting container was manu- \\ factured and tested under experimental conditions. Twelve Kato-Katz slides were performed as golden standard for \\ comparison. Quantitative results can be carried out by counting miracidia in a plate and parasite load can be calcu- \\ lated (miracidia/gram of feces). Statistically significant values were higher in the hatching test. More sensitive results, \\ with statistical significance, were achieved using $1.5 \mathrm{~g}$ of feces (which corresponds to 36 Kato-Katz slides) than by \\ using the Kato-Katz method. Advantages of this technique and its limitations are presented.
}

Key words: Schistosoma mansoni - diagnosis - feces

Accurate, inexpensive, rapid, and sensitive methods for diagnosis of human schistosomiasis mansoni on large populations and individual cases are imperative to control efforts, mainly based on treatment of infected people, sanitary engineering, health education, and snail control. Because of its relative simplicity and low-cost, the quantitative Kato-Katz fecal smear technique (Katz et al. 1972) is considered by the World Health Organization as the golden standard method for diagnosing this disease (WHO 1993). Nevertheless, low endemicity, post-treatment circumstances, or control of transmission can reduce worm burden and consequently decrease its sensitivity (Enk 2006).

Other approaches as immunologic methods could be more sensitive, but are more expensive and could present cross reactivity with other helminthic infections. These tests might not revert to negative after cure by chemotherapy. On the other hand, DNA-based detection technologies are very sensitive and specific, but are also very expensive and difficult to be performed in large scale, mainly under field conditions. A more sensitive parasitological methodology used as alternative is the hatching test for detection of miracidia.

Hatching methods are based on the strong positive phototropic behavior of the miracidia. It is interesting to note that Biomphalaria glabrata also presents positive phototropism. This synchronistic behavior certainly facilitates the finding of their intermediate hosts (Williams \& Coelho 1975) by the parasites. However, the lack of standardized procedures for performing the hatching test has led to a considerable variety of results. Recently, Yu et al. (2007) compared the Kato-Katz method (duplicate

\footnotetext{
+ Corresponding author: coelhopm@cpqrr.fiocruz.br Financial support: Fiocruz, $\mathrm{CNPq}$

Received 13 September 2007

Accepted 15 January 2008
}

$42 \mathrm{mg}$ fecal smears), the "nylon bag" hatching test (using $30 \mathrm{~g}$ of feces in each examination), and indirect hemagglutination assay to detect Schistosoma japonicum infection in endemic areas. They concluded that seven KatoKatz thick smears are equivalent to the performed hatching method, but emphasized the former technique is more practical and less difficult for preparation and examination.

In this report, a new hatching device is introduced and its efficiency and sensitivity are evaluated under experimental conditions, comparing with the golden standard Kato-Katz method. The present hatching test was devised to be very sensitive, rapid, and easy to perform for the detection of miracidia.

The miracidia hatching apparatus consisted of a regular 500-ml Erlenmeyer flask, adapted with a funnel in a small round collecting device (Fig. 4), as schematized in Fig. 1. A wooden box with a small aperture in its door was also developed to keep the apparatus in the dark during exposure of the collecting device to light (Figs 2, 3). A black rubber ring was used in the short connecting duct to prevent light from passing through the aperture in the wooden box during light exposure (Figs 2, 3). A bigger box for transport during field work was also manufactured (Fig. 5). In brief, when a schistosome-infected fecal suspension (or homogenised infected-tissue, for example liver or intestine) is placed in the flask, which is further placed in the dark, miracidia hatch and swim to the illuminated collecting container, being trapped in this compartment. The miracidia are easily recovered with a pipette.

To first test the efficacy of the apparatus, ten hamsters exposed to 100 cercariae of Schistosoma mansoni (LE strain) were killed after about 60 days. Their livers were chopped, homogenized, and left decanted into a sedimentation cone in the dark. The supernatant was removed by sucking through a pipette, whereas the sediment was resuspended in $20 \mathrm{ml}$ of cold water and poured into our apparatus. Before we make up the volume of the Erlenmeyer flask, the collecting container was first filled with dechlorinated-water for its superior opening with a pipette. The level of water reached the small hole at the funnel apex. Afterwards, dechlorinated water at $29^{\circ} \mathrm{C}$ was 

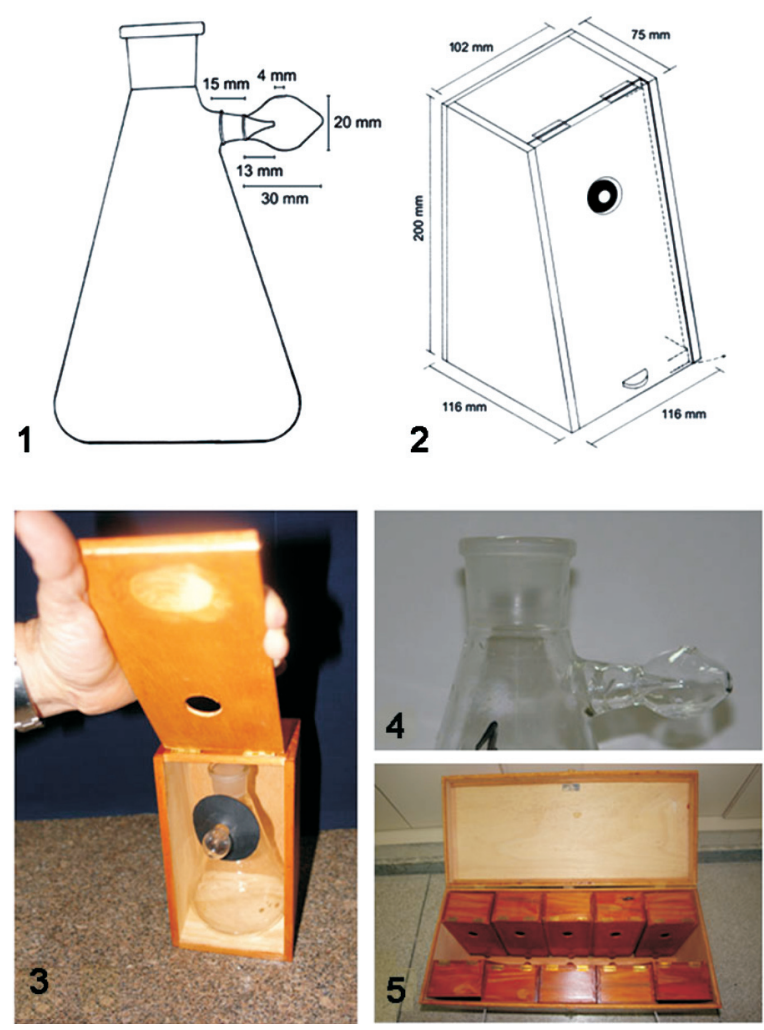

Figs 1-5: The miracidia hatching device. 1: lateral schematic drawing of the hatching flask; 2 : three-dimensional schematic drawing of the exposing wood box; 3 : the hatching flask inside the exposing box. Note the black rubber ring in the communicating duct; 4: detail of the upper part of the flask, showing the funnel; 5: the transportation box.

added to the flask, until reach the communicating level of both chambers. The flask was put inside the box, and the collecting container exposed to light. A wealth of swimming miracidia were readily observed by eye, which allows the inference that this method was very satisfactory for the attainment of a large biomass of larva.

To determine the sensitivity of the apparatus for stool examination and compare with the Kato-Katz method as golden standard, fresh stools from mice previously exposed to 100 cercariae of $S$. mansoni (LE strain) were collected from 50 days post-infection. Mouse stools were soaked in a small volume of cold dechlorinated water and filtered in gauze to decant into a sedimentation cone in the dark, in accordance with Hoffman, Pons and Janer (HPJ) method (Hoffman et al. 1934). The supernatant was removed by sucking with a pipette. The sediment was homogenized with human feces negative to $S$. mansoni, previously filtered in a $200 \mu \mathrm{m}^{2}$ mesh. At random, samples of $500 \mathrm{mg}$ were attained using a metal template with a central hole and placed in each hatching flask (one or three flasks per experiment) and twelve KatoKatz slides (42 mg of feces per slide, performing a total amount of $500 \mathrm{mg}$ ) were prepared for each experiment. In the hatching test, the collecting container was first filled with dechlorinated-water by pipetting and the fecal sample was left inside the flask, which was filled with water as described above. The flask was placed in the dark and the collecting container exposed to incandescent light for two hours. After this period, the miracidial suspension was collected by pipetting and left in an ice bath for $15 \mathrm{~min}$. The supernatant was discarded and the remaining volume (circa $2.0 \mathrm{ml}$ ) was aliquoted in a 12-well excavated plate (Kline's plate). A Lugol's iodine staining drop was added in each well and miracidia were counted under stereomicroscopy. Fourteen replicates were performed. Considering all fecal samples should be positive for schistosomiasis mansoni, sensitivity for both methods was calculated by the number of positive findings divided by the total number of experiments $(\mathrm{n}=11)$. The arithmetical mean number of miracidia per half gram of feces in the hatching test and the total number of eggs per half gram of feces in the Kato-Katz examinations were the final result. Statistical analysis (paired T-test) was performed considering significance level at $\mathrm{p}<0.05$.

The sensitivity of each method in this survey was $100 \%$ for the hatching test and $81.81 \%$ for 12 Kato-Katz slides. Considering the daily mean, the mean number of miracidia detected in the hatching test was $11.85 \pm 4.24$, while the mean number of eggs found in 12 Kato-Katz thick smears was $6.00 \pm 2.00$. The values were significantly higher in the new hatching test than in the KatoKatz method $(p=0.003)$. If considering the replicates $(\mathrm{n}=14)$, the number of miracidia was $12.80 \pm 4.02$, while the mean number of eggs was $6.43 \pm 2.14$. The values were also significantly higher $(\mathrm{p}=0.000)$. For control, the sediment in the bottom of the hatching flask was observed under brightfield microscopy in some experiments, and it was very difficult to find any non-hatched egg.

Aiming to increase the hatching test efficiency, we enlarged the fecal amount in each hatching flask to 1.5 and 3.0 g. Twelve Kato-Katz slides were also performed (circa $500 \mathrm{mg}$ ) for comparison. The number of miracidia significantly increased in the first group $(1.5 \mathrm{~g})$, but decreased in the latter group. In this system, it seemed that bigger amounts of feces than $1.5 \mathrm{~g}$ may inhibit miracidia ability to hatch and/or to swim toward the light source, probably because of the cloudy water. The fecal amount used in each flask $(1.5 \mathrm{~g})$ corresponds to 36 Kato-Katz thick smears, but the analysis and counting of miracidia lasted only 10 min per flask, while the microscopic survey lasted circa $2 \mathrm{~h}$ per 12 slides. In contrast to the KatoKatz method, the counting of miracidia does not require a well trained microscopist. In addition, in the same manner as the Kato-Katz technique, parasite load can also be inferred, but from the miracidia/g of feces ratio. Considering the miracidia as the infective form for snails, this could be an important epidemiological parameter on the study of schistosome transmission in the field.

Although Yu et al. (2007) reported inconsistent and only qualitative results in the analysis of $30 \mathrm{~g}$ of feces in the "nylon bag" hatching test, our experimental evaluation of this new hatching device indicates a high sensitivity and consistency. Probably the excessive amount of feces used by them, which could render the water cloudy, and the addition of a collecting container in our method could explain the discrepancy of the results. Miracidia stay concentrated in a small volume of limpid water, 
which facilitates the counting. Of course, hatching techniques have an obvious limitation, because of fecal samples must be fresh. To overcome this limitation, the same experimental design using $1.5 \mathrm{~g}$ of feces $(\mathrm{n}=10)$ was performed, but the collected miracidial suspensions were fixed in 10\% formol (1:4 parts of fixative) for 15 days. Fresh unfixed suspensions were examined for comparison. No significant difference was observed $(p=0.245)$. This finding allows an important improvement in the logistic design of epidemiological surveys, since attained suspensions can be fixed in the field and later examined in the laboratory.

In summary, we propose a simple and cheap hatching test with a collecting container for diagnosing schistosomiasis in individual or large scale investigations. This device has some clear advantages, because miracidia do not sink to the bottom of the flask with time like other hatching methods and stay concentrated in a small volume of limpid water. As the fecal sample is placed in a flask, diarrheic feces can be used. The sediment at the bottom of the Erlenmeyer flask can also be analyzed in the same manner as in HPJ method for evaluation of other helminth infections. Although a population screening is needed, this method is statistically more sensitive than Kato-Katz thick-smear under experimental conditions and makes possible the rapid examination of $1.5 \mathrm{~g}$ of feces, which corresponds to 36 Kato-Katz slides. Quantitative studies can be carried out, but if needed qualitative surveys can be performed using a simple magnifying glass. Suspensions obtained from the apparatus can be also fixed in $10 \%$ formol, with no significant lost of sensitivity. The device is also reusable as many times as necessary, and only dechlorinated water must be changed. Finally, we believe that this new hatching method could contribute for a more sensitive parasitological diagnosis of schisto- somiasis. Moreover, it works also as an excellent method to get miracidia free of host contaminants for immunohistochemistry, molecular, and biochemical studies.

\section{ACKNOWLEDGEMENTS}

To Anna Carolina Lima, from Instituto de Pesquisa René Rachou-Fiocruz, MG, for her assistance on biostatistics, Dr. Vania Dias, from Laboratório de Patologia, Instituto Oswaldo Cruz-Fiocruz, RJ, for schematic drawings of the hatching device, Mr. João de Araújo Dutra Filho, from DIRAC-Fiocruz, $\mathrm{RJ}$, for manufacturing the hatching device, Ms. Vera de Paula Ribeiro, from Laboratório de Esquistossomose, Centro de Pesquisa René Rachou-FIOCRUZ, MG, and Dr. John Kusel, from the Glasgow University, for English review.

\section{REFERENCES}

Enk MJ 2006. Avaliação da Infecção pela Esquistossomose mansoni em Áreas de Baixa Endemicidade com Enfoque no Diagnóstico, PhD Thesis, Centro de Pesquisa René Rachou, Fundação Oswaldo Cruz, Belo Horizonte, 85 pp.

Hoffman WA, Pons JA, Janer JL 1934. The sedimentation-concentration method in Schistosomiasis mansoni. PR J Public Health Trop Med 9: 283-291.

Katz N, Chaves A, Pellegrino J 1972. A simple device for quantitative stool thick-smear technique in schistososimiasis mansoni. Rev Inst Med Trop São Paulo 14: 397-400.

WHO - World Health Organization 1993. The control of schistosomiasis. Second report of the WHO Expert Committee, WHO Tech Rep Ser 830, Geneve.

Williams P, Coelho PMZ 1975. Atraction of snail to betalights. Trans R Soc Trop Med Hyg 67: 616-617.

Yu JM, de Vlas SJ, Jiang QW, Gryssels B 2007. Comparison of the Kato-Katz technique, hatching test and indirect hemagglutination assay (IHA) for the diagnosis of Schistosoma japonicum infection in China. Parasitol Int 56: 45-49. 